\title{
Time-synchronized Data Collection in Smart Grids through IPv6 over BLE
}

\author{
Stanley Nwabuona \\ Pro2Future GmbH \\ Graz, Austria \\ stanley.nwabuona@pro2future.at
}

\author{
Simon Mayer \\ Pro2Future GmbH and Graz \\ University of Technology \\ Graz, Austria \\ simon.mayer@pro2future.at
}

\begin{abstract}
For the operation of electrical distribution system an increased shift towards smart grid operation can be observed. This shift provides operators with a high level of reliability and efficiency when dealing with highly dynamic distribution grids. Technically, this implies that the support for a bidirectional flow of data is critical to realizing smart grid operation, culminating in the demand for equipping grid entities (such as sensors) with communication and processing capabilities. Unfortunately, the retrofitting of brown-field electric substations in distribution grids with these capabilities is not straightforward - this scenario requires a solution that provides "industry-grade" Internet of Things capabilities at "consumer-grade" prices (e.g., off-the-shelf communication standards and hardware). In this paper, we discuss the particular challenge of precisely timesynchronized wireless data collection in secondary substations that at the same time supports on-site configuration by authorized maintenance personnel through a mobile application: to achieve this, we propose a combined implementation of IPv6 over Bluetooth Low Energy.
\end{abstract}

\section{ACM Classification Keywords}

C.2.1. Network Architecture and Design: Wireless communication

\section{Author Keywords}

Time-synchronization; Wireless Communication; Dependable IoT; Smart Grid Automation.

\footnotetext{
Permission to make digital or hard copies of part or all of this work for personal or classroom use is granted without fee provided that copies are not made or distributed for profit or commercial advantage and that copies bear this notice and the full citation on the first page. Copyrights for third-party components of this work must be honored. For all other uses, contact the owner/author(s).

IOT '18 October 15-18, 2018, Santa Barbara, CA, USA

(C) 2018 Copyright held by the owner/author(s).

ACM ISBN 978-1-4503-6564-2/18/10

DOI: https : //doi .org/10.1145/3277593. 3277632
}

\section{INTRODUCTION}

Due to the progressing integration of distributed energy resources (such as domestic photovoltaics) into the distribution grid [5], conventional - mostly passive - monitoring and control schemes of power systems are no longer applicable. This is because these are not designed to handle and manage the volatile and dynamic behavior stemming from these new active grid entities. Therefore, advanced controlling and monitoring schemes are required for distribution grid operation (i.e., including in Low-Voltage (LV) grids) to avoid system overload which could incur permanent damages.

A possible solution to this problem is to extend the conventional SCADA (Supervisory Control and Data Acquisition) systems to the lower voltage levels. However, the deployment of such systems in the LV grid is costly since a lot of brown-field equipment needs to be retrofitted (e.g., a city like Vienna features around 12000 transformer stations with 5-9 measurement points each). Consequently, an ideal solution is envisaged to provide a cost-efficient monitoring and control of hardware and software, through communication technologies that integrate the hardware with backends both locally (e.g., a Programmable Logic Controller (PLC) within a substation) and remotely (e.g., a cloud-based SCADA system) [2].

For many grid monitoring scenarios, communication between nodes is geared towards the ability to perform three basic operations [1]: generation scheduling; automatic generation control; and special protection schemes. Generation Scheduling enforces the optimized use of power resources by either committing enough generating units during load peaks or turning them off at load troughs. Automatic Generation Control maintains system frequency by tracking load variations while Special Protection Schemes provide reliability through timely detection of system anomalies and implementation of corrective measures. These operations impose a set of requirements on a communication network in order to be realized. For instance, many protection schemes define boundaries in packet propagation delays as well as synchronization requirements 


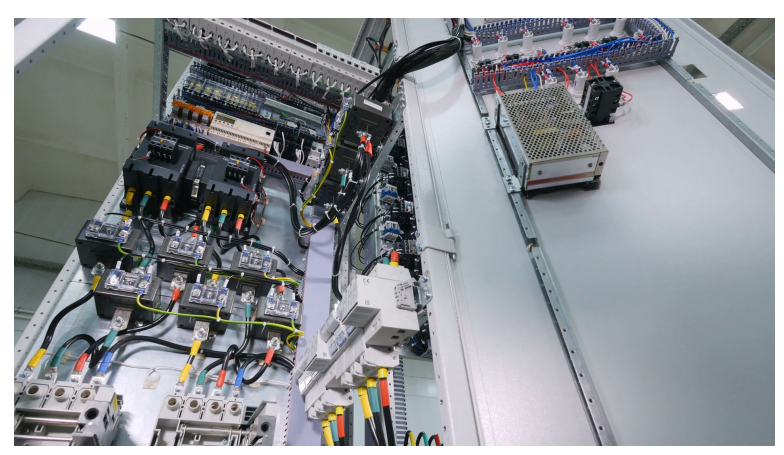

Figure 1. A transformer substation in the $\mathrm{LV}$ grid.

on the data collection (i.e., collected data values from different sensor nodes must be time-synchronized within, e.g., 20 $\mu$ s).

While it is rather straightforward to satisfy such requirements when using wired communication technologies such as Ethernet and their companion standards (e.g., IEEE 1588 for time synchronization), the deployment overhead of any system that requires additional wires inside a brown-field substation is prohibitive: often, dozens of sensors are required in a substation, leading to dozens of additional wired connections in an already crowded environment (see Fig. 1 for an example). Furthermore, although Power Line Communication technologies would trivialize the retrofitting regarding communication, it is unreliable due to the indeterminate nature of impedance variations on the grid lines and cross-talk - these factors strongly affect the performance of these protocols in realistic settings: in our tests in deployed electrical grids, it was possible to transmit a measurement reliably only every $2.5 \mathrm{~min}$. On the other hand, reliable wireless networks would allow for relatively low deployment overhead in these settings - however, Quality of Service (QoS) parameters of these systems are usually inferior compared to wired designs.

In this paper, we discuss the possibility of using wireless technologies for time-synchronized data collection in electric substations in the LV grid and with respect to realistic context parameters and requirements. We start by discussing these requirements, which were derived together with one of our industry partners, Siemens AG, a leading global supplier of solutions and services for electrical transmission and distribution; from these requirements, we derive concrete design criteria (see Section 2). Next, we derive and discuss options of how the requirements might be satisfied (see Section 3). We describe our final design and the steps we take toward its implementation and deployment in Section 4 before providing a brief conclusion.

\section{APPLICATION REQUIREMENTS}

Together with our industry partner, we have derived the following four major requirements on time-synchronized data collection networks of sensor nodes that are deployed in secondary substations in the LV grid:

- Industry Standard Hardware: The developed system should be deployed on a microcontroller from the Texas Instruments (TI) CC26xx SimpleLink family such as the
$\mathrm{CC} 2650^{1}$. This requirement stems from the long battery lifetime offered by these chips when operated on coin cell batteries, their applicability in the context of energy-harvesting applications, and TI's position as a trusted partner for large industrial companies.

- Precise Time-synchronization: Our targeted scenario (implementation of a smart grid protection scheme) is dependent on the synchronized collection of data from several sensor nodes within a secondary substation. In particular, to derive valid phasor measurements, our sink node is required to precisely match zero crossings of the measured electrical waves from multiple PMUs (Phasor Measurement Units) with an acceptable accuracy in the order of $\mu$ s [3].

- Minimal Deployment and Maintenance Effort: With electric utilities as target-users, an optimal solution should minimize users' CAPEX (Capital Expenditure) and OPEX (Operational Expenditure). This objective can be achieved by employing low-cost and commercial off-the-shelf (COTS) hardware and minimizing the amount and heterogeneity of deployed components as well as the power consumption of the equipment for measurement and communication.

- Hand-held Reconfiguration: The deployed system is required to support the simple reconfiguration of individual sensor nodes by maintenance personnel. In particular, it should be possible to reconfigure nodes using a COTS handheld device such as a smartphone or tablet.

\section{IMPLEMENTATION OPTIONS}

A basic synopsis of the previous section is a low-cost system which can be readily retrofitted into a brown-field substation while enabling data collection that supports basic operations of a smart grid. The use of TI CC2650 MCUs supports a low-cost system and its low-power mode makes the system capable of running on energy-harvesting only: experiments by our industry partner showed that CC2650-based sensor nodes are indeed able to harvest sufficient energy inside a secondary substation to sustain the transmission of a Bluetooth Low Energy (BLE) packet every 60-90 seconds. Besides cost minimization, support for energy-harvesting improves the viability of the entire system in a brown-field deployment by trivializing the need for extra power supply outlets for the nodes. Furthermore, wireless technologies support the communication with maintenance personnel if they can interface with the BLE interfaces of COTS hand-held devices.

The remaining challenge is thus associated with the requirement for precise time synchronization. To address this, we considered the following options:

- Dedicated Time-synchronization Component: In order to support the two modes of radio operation, a dedicated, secondary radio can be used. For this a simple and deterministic radio chip such as the TI CC2520 $(2.4 \mathrm{GHz})$ or TI CC1200 (sub GHz) can be used. Other silicon vendors also provide similar but often even cheaper solution such as the Nordic Semiconductors nRF24L01+ (2.4GHz) or nRF905

\footnotetext{
$\overline{1_{\text {WWW . ti . com/product/cc2 } 650}}$
} 
(sub GHz). However, these often use proprietary protocols such as Shockburst. Overall this approach adds further cost as well as additional components such as crystal oscillators that may malfunction. Depending on the frequency used for this secondary radio there needs to be arbitration between the radio. Overall the complexity of the solution drastically increases and there is often little to no support for multiple, concurrent radio interfaces in RTOS for constrained devices.

- Optical/Acoustic Out-of-band Synchronization: As the sensor nodes and the border router typically share are closed of space, it is possible to add an optical transceiver. There are two main categories: (i) visible light communication (VLC) as standardized by IEEE 802.15.7 or open-source project such as OpenVLC); (ii) non-visible light communication such as infrared Data Association IRDA. Either approach heavily depends on a line of sight however and is prone to dust and dirt blocking the signal. Similarly Acoustic transceivers could be to transmit signals either audible or ultra-sonic, the limited speed of sound makes synchronization at the required precision complex if not impossible. As with the dedicated radio solution there is only limited support in operating systems while also increasing the number of components and cost of the solution.

- Glossy-based Flooding: Glossy is a flooding protocol for wireless sensor networks that enables fast network flooding and implicit precise time synchronization [4]. Glossy and derivative protocols can achieve the level of precision in time synchronization by design as it is required for constructive interference. However since the initial inception of the protocol only the original CC2420 has been fully supported. The reason for this is that a very precise control of the radio - ideally at a register level - is required, and that the timing of sending and receiving needs to be very deterministic. There is a port for the TI CC2538; however, like the original TI CC2420, this chip does not support Bluetooth Classic nor Low Energy hence a dedicated BLE radio would be required. Multi-protocol SoC's such as the CC2650 employ a secondary processor (called the RF core) to perform the actual transmission and reception of packets. This RF core is then connected via a mailbox to the application processor. Due to this complex and possibly non deterministic architecture, no port of Glossy for such a device exists and may never exist at all. Therefore, as with all previous solutions, the separation into a BLE and 802.15.4 radio would increase complexity and cost of the solution.

- Precision Time Protocol (PTP): The Zephyr RTOS ${ }^{2}$ has support PTP (IEEE 1588) on its roadmap (1.13 release). Although PTP promises time synchronization on a sub$\mu$ s level, this is currently only possible on specific Ethernet chips that support IEEE 802.1 TSN/AVB (which timestamps packets on arrival). As wires are not an option for the sensor nodes this approach cannot be used. Select WiFi chips such as the TI WL8 series support a similar, albeit non standard approach of time-stamping packets in the radio itself. In addition to the high power consumption of the radio

\footnotetext{
${ }^{2}$ https://www . zephyrproject.org/
}

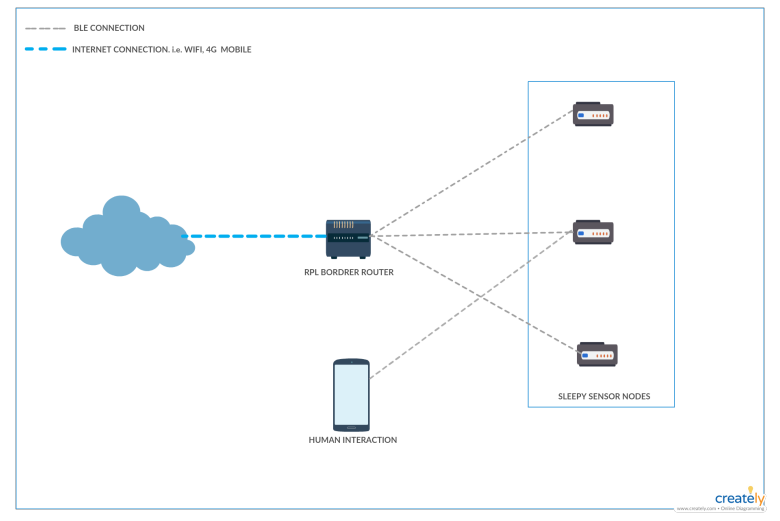

Figure 2. Network Layout

itself, a full Linux OS is currently required. This makes it unsuitable for a devices powered by energy harvesting.

- Dual-Protocol Stack: Many modern SoC with integrated radio such as the TI CC13xx/CC26xx series, Nordic Semiconductors nRF52840, Silicon Labs Mighty Gecko or NXP KW41 series support dual-stack operation. While this increases the complexity of the SoC, the software provided by the chip vendor usually handles this complexity. Some vendors already include a radio arbiter allowing concurrent operation of multiple protocols. Open-source solutions such as BLEach, a connection-oriented IPV6-over-BLE stack[6], leverages the wide adoption of BLE standards to improve interoperability across multi-vendor devices. Thanks to the low-level access to the network stack in BLEach, timestamping can be implemented on the MAC layer allowing low- $\mu$ s time synchronization.

\section{SYSTEM ARCHITECTURE}

Based on the deliberations of the previous section, we propose a Wireless Sensor Network (WSN) whose architecture is based on the TI CC2650 for low-cost, seamless brown-field deployment and support for dual protocol. Also, we introduce BLEach which leverages on the hardware's support for dual protocols to encourage heterogeneous network communication as well as time synchronization.

The proposed network will be implemented in a star topology (See Figure 2) comprised of three TI CC2650 Launchpads running the Contiki OS. Three of these Launchpads will programmatically emulate the distribution grid sensors which are hall effect energy harvesters. A RaspberryPi with 6LoWPAN support will serve as the router, performing a stateless autoconfiguration through Router Advertisement messages. The sensor nodes are the clients and shall synchronously send data every 1.5 minutes, unless interrupted by a button press signaling the TI CC2650 sensor node to switch over to the pure BLE Stack for hand-held configurations.

The implemented system will be validated and tested in a laboratory setup (see Fig. 2), in particular regarding its timesynchronization precision. Subsequently, we are planning to deploy the complete system as part of the smart grid test-bed 
of the Aspern Smart City Research campus in Vienna, Austria. This test-bed comprises several distribution grids equipped with metering devices and secondary substations that we can access and retrofit with the proposed system.

\section{Conclusion}

Today's and future power grids require more active management, which is dependent on reliable monitoring and control equipment that can satisfy industry-grade requirements but is still cost-effective to deploy and operate. In this paper, we discussed a specific challenge: equipping brown-field distributed substations in the LV-grid with a monitoring solution that enables time-synchronized data collection, works with current industry hardware, enables on-site reconfiguration through hand-held devices, and remains deployable and maintainable in realistic settings. We proposed to create a reliable, timesynchronized wireless network on top of the BLEach protocol. This solution has the added benefit of a high cost-efficiency potential, as it can be deployed as well on the (cheaper) TI CC2640 MCUs $^{3}$ as well. In addition, by seamlessly integrating with BLE clients, this solution supports the on-site (re)configuration of sensor nodes.

\section{Acknowledgements}

The presented work was funded and supported by the Austrian Research Promotion Agency (FFG) (\#854184 COMET) and the Austrian Climate and Energy Fund (KLIEN).

\section{REFERENCES}

1. Dhananjay M. Anand, Jeffrey G. Fletcher, Ya-Shian Li-Baboud, Julien Amelot, and J. A. Moyne. 2010. Using clock accuracy to guide model synthesis in distributed systems: An application in power grid control. In 2010 IEEE International Symposium on Precision Clock Synchronization for Measurement, Control and Communication (ISPCS 2010). 7-12.

2. Konrad Diwold, Simon Mayer, Alfred Einfalt, Josiane Xavier Parreira, Jack Hodges, and Darko Anicic. 2017. Grid Watch Dog: A Stream Reasoning Approach for Lightweight SCADA Functionality in Low-Voltage Grids. In Proceedings of the 8th International Conference on the Internet of Things (IoT 2018). to appear.

3. Atis Elsts, Simon Duquennoy, Xenofon Fafoutis, George Oikonomou, Robert Piechocki, and Ian Craddock. 2016. Microsecond-Accuracy Time Synchronization Using the IEEE 802.15.4 TSCH Protocol. In 2016 IEEE 41st Conference on Local Computer Networks Workshops (LCN Workshops). 156-164.

4. F. Ferrari, M. Zimmerling, L. Thiele, and O. Saukh. 2011. Efficient Network Flooding and Time Synchronization with Glossy. In Proceedings of the 10th ACM/IEEE International Conference on Information Processing in Sensor Networks. 73-84.

5. Stefano Rinaldi, Davide Della Giustina, Paolo Ferrari, Alessandra Flammini, and Emiliano Sisinni. 2016. Time Synchronization over Heterogeneous Network for Smart

\footnotetext{
3http: //www . ti . com/product/CC2640
}

Grid Application. Ad Hoc Netw. 50, C (Nov. 2016), 41-57.

6. Michael Spörk, Carlo Alberto Boano, Marco Zimmerling, and Kay Römer. 2017. BLEach: Exploiting the Full Potential of IPv6 over BLE in Constrained Embedded IoT Devices. In Proceedings of the 15th ACM Conference on Embedded Network Sensor Systems (SenSys '17). ACM, New York, NY, USA, Article 2, 14 pages. 